\title{
Efecto del recubrimiento comestible a base de almidón de yuca sobre los parámetros químicos y sensoriales de cascos de guayaba
}

\author{
Effect of edible coating based on cassava starch \\ on the chemical and sensory parameters of guava \\ hulls
}

Susmara Amaiz Mota Universidad de Oriente, Núcleo Monagas Estado Monagas - Venezuela

Julio Colivet Universidad de Oriente, Núcleo Monagas Estado Monagas - Venezuela

Adolfo Cañizares Instituto Nacional de Investigaciones Agrícolas (INIA) - Estado de Monagas - Venezuela

Revista Cumbres Vol.5 №1

Versión impresa ISSN 1390-9541

Versión electrónica ISSN 1390-3365

http://investigacion.utmachala.edu.ec/revistas/index.php/Cumbres 


\section{RESUMEN}

La guayaba es un fruto que sigue respirando y transpirando luego de ser cosechada ocasionando el marchitamiento, flacidez, pérdida de peso, cambios en el color entre otros, disminuyendo a la vez su calidad y por ende su valor comercial. Para reducir estos cambios se vienen utilizando métodos de conservación como las atmósferas modificadas o controladas que combinadas con la refrigeración han logrado conservar por más tiempo las características de calidad de los frutos. La tendencia a nivel mundial que permite conservar estas características es el de los recubrimientos comestibles. El objetivo de este estudio es evaluar la calidad química y sensorial de cascos de guayaba (cultivar cubana suprema roja) sometidos a recubrimientos comestibles con distintas concentraciones de almidón de yuca (0, 5 y 7\%) y glicerina como plastificante (5\%), y almacenamiento durante 27 días en refrigeración a 8 ${ }^{\circ} \mathrm{C}$, los parámetros fueron evaluados cada 3 días durante el almacenamiento. Los resultados mostraron que el $\mathrm{pH}$, SS, AT, y Vit. C, tuvieron menor variación con las formulaciones 2 y 3. La evaluación sensorial a través del panel semientrenado arrojó resultados favorables en los 3 parámetros estudiados (aroma, consistencia y sabor) para las muestras con recubrimientos comestibles con la formulación 3. En tal sentido queda evidenciado que la aplicación de recubrimientos comestibles a base de almidón de yuca y glicerina se puede considerar como un método alternativo para alargar la vida útil de frutas, sin alterar sus características químicas originales y manteniendo una aceptabilidad organoléptica favorable.

Palabras clave: Recubrimientos comestibles, guayaba, almidón de yuca.

\section{ABSTRACT}

The guava is a fruit that continues to breathe and perspire after being harvested causing wilting, sagging, weight loss, changes in color among others, decreasing both its quality and therefore its commercial value. To reduce these changes, conservation methods have been used, such as modified or controlled atmospheres that, combined with refrigeration, have managed to preserve the quality characteristics of the fruits for longer. The worldwide tendency to preserve these characteristics is that of edible coatings. The objective of this study is to evaluate the chemical and sensory quality of red supreme banana guava hulls subjected to edible coatings with different concentrations of cassava starch (0,5 and 7\%) and glycerin as plasticizer (5\%) and stored during 27 days in refrigeration at $8{ }^{\circ} \mathrm{C}$, the parameters were evaluated every 3 days during storage. The results showed that $\mathrm{pH}, \mathrm{SS}, \mathrm{AT}$, and Vit. C, had less variation with the formulations 2 and 3 . The sensory evaluation through the panel semilibnado gave favorable results in the 3 parameters studied (aroma, consistency and taste) for samples with edible coatings with the formulation 3. In this sense is evidenced that the application of edible coatings based on cassava starch and glycerin can be considered

\section{Cumbres}


as an alternative method to extend the useful life of fruits, without altering their original chemical characteristics and maintaining a favorable organoleptic acceptability.

Keywords: Edible coatings, guava, cassava starch.

\section{INTRODUCCIÓN}

El cultivo de la guayaba (Psidium guajava L.) ocupa el tercer lugar en el país en cuanto al área sembrada y el sexto por el valor de la cosecha, después de los cítricos, la piña y el mango (Mata y Rodríguez, 1990). Desde el punto de vista nutricional, la guayaba es una de las frutas con mayor contenido de vitaminas (A y C), minerales (fósforo y calcio) y proteínas. Además, es muy atractiva para su consumo diario en cualquier forma (fruta fresca o procesada en bocadillos, jalea, néctar, en cascos o jugos).

En la actualidad se ha aumentado el consumo de frutas, lo cual representa un beneficio para los productores. Sin embargo, hay que tomar en cuenta el problema que estas siguen respirando y transpirando luego de ser cosechadas ocasionando el marchitamiento, flacidez, pérdida de peso, cambios en el color entre otros, disminuyendo a la vez su calidad y por ende su valor comercial. Para reducir estos cambios se vienen utilizando métodos de conservación como las atmósferas controladas y atmósferas modificadas que combinadas con la refrigeración han logrado mantener por más tiempo las características de calidad de los frutos. Sin embargo, estas técnicas presentan limitaciones como: los altos costos e instalaciones especiales, así como el uso de polímeros poco biodegradables que causan gran impacto ambiental. Estos inconvenientes han despertado el interés de los investigadores en la búsqueda de soluciones a menor costo e impacto ambiental.

Uno de los métodos que permite preservar las características originales de las frutas cortadas, es el recubrimiento comestible, el cual es una capa delgada de material comestible formado como un revestimiento sobre el alimento (McHugh y Senesi, 2000).

Por otro lado, Martín et al. (1992) definieron a los recubrimientos comestibles como capas delgadas de materiales los cuales pueden ser ingeridos por el consumidor, que proveen una barrera al transporte de masa a través del alimento fresco o manufacturado. Paralelamente, Carrasco et al. (2002) manejaron un concepto que fusiona las dos definiciones anteriores: los recubrimientos comestibles son capas delgadas de un material biopolímero (proteína o polisacárido como una solución hidrocoloide o como una emulsión con lípidos) que son aplicadas sobre la superficie de un alimento en adición o reemplazo de la corteza natural y que se comportan principalmente como barreras que reducen la difusión de gases $\left(\mathrm{O}_{2}, \mathrm{CO}_{2}\right.$, vapor de agua), permitiendo extender la vida útil del alimento.

Estos recubrimientos han sido desarrollados con el fin de extender la vida útil de los productos alimenticios o enriquecerlos. Pueden usarse como 
soporte de agentes antimicrobianos, antioxidantes o nutrientes tales como vitaminas y minerales, como portadoras de otros aditivos o para retardar la migración de humedad y lípidos. Su manipulación no debe producir un deterioro en el producto y, además, deben ser totalmente neutros con respecto al color, tacto y olor del alimento (Martín et al., 1992).

Los almidones son hidrocoloides comúnmente utilizados para la formulación de estos recubrimientos, principalmente el almidón de yuca por su alto rendimiento y su fácil adhesividad al alimento, además de aportar un brillo atractivo al recubrimiento. Junto con ellos, es habitual e indispensable la adición de un plastificante como glicerina para mejorar la flexibilidad del recubrimiento comestible.

En el caso particular de frutas y hortalizas para consumo en fresco, los recubrimientos comestibles proporcionan una cubierta protectora adicional cuyo impacto tecnológico es equivalente al de una atmósfera modificada, por lo tanto, representan una alternativa a este tipo de almacenamiento ya que es posible reducir la cinética de los cambios de calidad a través de la modificación y control de la atmósfera interna en estos productos vegetales.

El objetivo de este estudio es evaluar la calidad química y sensorial de cascos de guayaba (cultivar cubana suprema roja) sometidos a recubrimientos comestibles con distintas concentraciones de almidón de yuca.

\section{MATERIALES Y MÉTODOS}

\section{Materia prima}

Los frutos de guayaba (Psidium guajava L. cv. Cubana suprema Roja) se obtuvieron en Caicara, municipio Cedeño, estado Monagas ubicado a una altitud de $200 \mathrm{msnm}$, con precipitación promedio de $1068 \mathrm{~mm}$ y temperatura promedio de $26^{\circ} \mathrm{C}$; la cosecha se realizó en época seca. El criterio de selección se basó en una inspección visual, donde los frutos no presentaron daños mecánicos, plagas, enfermedades u otros defectos. Los frutos tuvieran un tamaño homogéneo y en madurez fisiológica de 80-20\%, la cual se identificó por el color de la piel (epicarpio) (80\% color amarillo y 20\% coloración verde, grado 3 en la escala de colores de maduración de la guayaba). Las yucas que se utilizaron para la extracción del almidón se obtuvieron en el mismo sitio, las raíces cosechadas tenían edad de 9 meses, se seleccionaron las que no presentaran daños mecánicos, plagas, enfermedades u otros defectos.

\section{Preparación de la muestra de guayaba}

Seleccionados los frutos, se procedió a realizar un lavado a mano con abundante agua potable para remover la suciedad, partículas extrañas y reducir el contenido de microorganismos. Seguidamente se dejaron escurrir hasta eliminar el exceso de agua en la superficie, para luego ser sometidos a un proceso de desinfección con una solución clorada (100ppm de hipoclorito de sodio) durante dos minutos. Para la obtención de los cascos de guayaba se

\section{Cumbres}


realizó un corte por la mitad (con un cuchillo de acero inoxidable para evitar la oxidación del fruto), se extrajeron las semillas con una cucharilla y se eliminó la piel (epicarpio) con un pelador de acero inoxidable.

\section{Obtención del almidón de yuca}

El proceso se realizó de forma manual, se procedió al lavado de la raíz de yuca con abundante agua potable para eliminar los restos de suelo, impurezas y partículas extrañas. El pelado de las raíces se realizó con un cuchillo de acero inoxidable para evitar la oxidación de la pulpa, posteriormente se sumergió en solución de hipoclorito de sodio (100 ppm) durante 10 minutos. Transcurrido el tiempo, la pulpa fue cortada en trozos pequeños aproximadamente de $4 \mathrm{~cm}$ de diámetro y $2 \mathrm{~cm}$ de espesor, luego se procesaron los trozos de yuca en una licuadora industrial en una proporción de 1:3 (por cada kilo de pulpa de yuca, tres litros de agua). Una vez obtenida esta solución se procedió al filtrado a través de una tela de filtrado ejerciendo presión para extraer la mayor cantidad de líquido posible. La suspensión obtenida del filtrado se dejó en decantación por 24 horas en un recipiente tapado y en refrigeración $\left(10^{\circ} \mathrm{C} \pm 2^{\circ} \mathrm{C}\right)$. Transcurrido ese tiempo, se retiró el sobrenadante con cuidado para no remover el almidón y el sedimento que quedó se expandió en una bandeja de acero inoxidable y se llevó a secado en una estufa de aire forzado a 55을 $\mathrm{C}$ durante 12 horas. Culminado el tiempo de secado, se retiraron las bandejas de la estufa y se dejaron enfriar durante 15 minutos a temperatura ambiente $\left(26+2{ }^{\circ} \mathrm{C}\right)$, para luego realizar el acondicionamiento, es decir, la molienda en un molino de martillo marca Wiley el cual trabaja a $1200 \mathrm{~mm} /$ seg con un tamiz de $5 \mathrm{~mm}$ y finalmente el almacenado en un frasco de vidrio herméticamente cerrado para evitar la absorción de humedad del ambiente.

\section{Elaboración de los recubrimientos comestibles}

Para la obtención de los recubrimientos comestibles se siguió la metodología descrita por Famá et al. (2008) con algunas modificaciones. Se prepararon tres formulaciones, utilizando el almidón de yuca como componente principal, se homogeneizaron en una suspensión acuosa de almidón de yuca, glicerina como plastificante y agua destilada durante 45 min, con agitación controlada y a temperatura ambiente $\left(20^{\circ} \mathrm{C}\right)$. Luego, se calentaron las soluciones a una velocidad de calentamiento de aproximadamente $1,8{ }^{\circ} \mathrm{C} / \mathrm{min}$, hasta $82^{\circ} \mathrm{C}$. Se tomó la temperatura final del proceso como aquélla que fuera 5\% mayor que la temperatura de gelatinización del almidón.

\section{Aplicación de los recubrimientos comestibles}

Los recubrimientos comestibles obtenidos fueron aplicados sobre los frutos de guayaba cortadas (cascos) según la metodología empleada por Vicentini et al. (1999). Los cascos de guayaba fueron separados en 3 lotes (A, B, y C), conformados por un peso estándar de fruta cortada para cada uno, los cuales fueron sometidos a inmersión en sus respectivos recubrimientos comestibles 
(0, 5 y 7 \% de almidón más glicerina, respectivamente). Los cascos de guayaba fueron sumergidos durante 5 min en las formulaciones: lote A: $0 \%$, lote B: 5\% y lote C: 7\% de almidón respectivamente, ejerciendo una ligera presión sobre ellos para evitar que flotaran en la superficie del recubrimiento. Seguidamente, los cascos fueron colocados en bandejas y se llevaron a secado en una estufa de aire forzado a $25 \stackrel{\circ}{\circ} \mathrm{C}$ durante $15 \mathrm{~min}$. Posteriormente, fueron llevados a una balanza analítica marca Kern modelo 770-14, para tomar el peso inicial de los cascos recubiertos.

\section{Almacenamiento}

Los lotes A, B y C fueron almacenados en bolsas plásticas de polietileno de baja densidad tipo Ziploc con cierre hermético, a temperatura de refrigeración de $8 \stackrel{\circ}{\circ}$. Los días $0,3,6,9,12,15,18,21,24$ y 27 se determinaron por triplicado para cada lote los parámetros químicos ( $\mathrm{pH}$, sólidos solubles, acidez titulable y vitamina C) y sensoriales (aroma, consistencia y sabor).

\section{Determinación de pH}

El pH se determinó con un potenciómetro marca "ORION" siguiendo el método descrito por Gallo (1996), para esto se obtuvo el zumo de los cascos de guayaba, del cual se tomaron $10 \mathrm{~mL}$ con una pipeta volumétrica y fueron colocados en un beaker de $100 \mathrm{~mL}$, se homogeneizó hasta que quedó libre de grumos. El potenciómetro fue calibrado con una solución buffer de $\mathrm{pH} 4$ y 7, se colocó en la muestra contenida en el vaso precipitado durante $15 \mathrm{~s}$ y posteriormente se registró la lectura indicada por el equipo.

\section{Determinación de sólidos solubles, SS (ㅂrix)}

Para determinar los SS expresados en Brix, se empleó el método de refractometría, según la norma COVENIN 924-83 (1983) para lo cual se utilizó un refractómetro modelo ABBE Operador Manual. La temperatura del refractómetro se mantuvo a $20{ }^{\circ} \mathrm{C}$, el equipo se calibró utilizando agua destilada antes de cada lectura.

\section{Determinación de acidez titulable (AT)}

Este parámetro se evaluó por medio de la norma COVENIN 1151-77. La acidez se determinó por titulación con una solución $0,1 \mathrm{~N} \mathrm{NaOH}$ y fenolftaleína al 1\% como indicador. Se anotó el volumen gastado de $\mathrm{NaOH}$ para el cálculo de la acidez titulable mediante la ecuación:

$$
\% A T=\frac{20 \times V 1 \times M E \times N}{3 V} \times 100
$$

\section{Cumbres}


Donde:

\%AT=Acidez titulable en gramos del ácido predominante (ácido cítrico) por $100 \mathrm{~g}$.

V= Volumen de la alícuota tomada para el análisis en mililitros.

V1 = Volumen de la solución de hidróxido de sodio empleado en la titulación, en mililitros.

$\mathrm{N}=$ Normalidad de la solución de hidróxido de sodio.

$\mathrm{ME}=$ Masa miliequivalente del ácido (ácido cítrico).

\section{Determinación de vitamina C (Vit. C)}

El contenido de vitamina $\mathrm{C}$ (\% ácido ascórbico) se determinó mediante maceración de la muestra con ácido oxálico (AO) y ácido clorhídrico (AC) (estabilizadores) y titulación con 2,6-Dicloroindofenol (2,6-DIF), como lo plantea Miller (2003). Se pesó 2,50 g de muestra en un beaker de $50 \mathrm{~mL}$, y se le adicionó $9 \mathrm{~mL}$ de $\mathrm{AO}$ y $1 \mathrm{~mL}$ AC; se dejó en reposo protegido de la luz y el oxígeno por 10 minutos. Transcurrido el tiempo se transfirió cuantitativamente a un balón aforado de $100 \mathrm{~mL}$ y se aforó con agua destilada. Se procedió a filtrar a través de tela de filtrado en un embudo, se tomó una alícuota de $10 \mathrm{~mL}$ del filtrado que fue colocado en erlenmeyer de $125 \mathrm{~mL}$ y se le adicionaron 9 $\mathrm{mL}$ de AO y $1 \mathrm{~mL}$ AC; procediendo a titular con 2,6-dicloroindofenol hasta la aparición de una coloración rosa pálido persistente por aproximadamente 5 s. Paralelamente se determinó el título o factor (F) mediante la preparación de una solución de ácido ascórbico 0,05 g/mL. Titulando alícuotas de $10 \mathrm{~mL}$ (añadidas de $9 \mathrm{~mL}$ de $\mathrm{AO}$ y $1 \mathrm{~mL} \mathrm{AC}$ ) contra 2,6-dicloroindofenol. El contenido de ácido ascórbico (AA) se reportó en mg/100g de muestra con la expresión:

$$
m g A A / 100 g=\frac{(F \times V m l(2,6-D I F) \times \text { Dilución }}{(\text { Peso } M \times \text { Alícuota })} \times 100
$$

Donde:

Mg AA: es la cantidad de ácido ascórbico contenidos en 100 g de muestra. F: es el factor o título.

V: es el volumen gastado de 2,6 (DIF) en la titulación expresado en mL.

\section{Evaluación sensorial}

Las características sensoriales (aroma, consistencia y sabor) de los cascos de guayaba con los recubrimientos comestibles fueron estudiadas a través de una planilla de deterioro de frutas de 5 puntos, que fue diseñada para evaluar los atributos de calidad sensorial de guayabas y cuyas características fueron establecidas, de acuerdo a los trabajos de Romojaro et al. (1996) y Aleixandre (1987). Antes de realizar el estudio se procedió a semientrenar un panel sensorial integrado por panelistas de ambos sexos, en edades comprendidas entre 22 y 40 años que consuman guayaba fresca, con conocimientos básicos de evaluación sensorial de los alimentos y los cuales recibieron 
un entrenamiento previo con muestras de guayaba fresca, para evaluar los atributos (aroma, consistencia y sabor), de acuerdo a la tabla establecida de 5 puntos. El entrenamiento del panel se realizó a través de pruebas de diferencia como la prueba de Duo- Trio y Triángulo.

\section{Diseño estadístico}

Se utilizó un diseño estadístico de bloques completamente al azar con arreglo de tratamiento factorial (3x10), (efecto del recubrimiento x efecto tiempos de medición de las variables químicas y sensoriales), los recubrimientos fueron 0,5 y 7\% de almidón de yuca y los tiempos de medición fueron 0, 3, 6, $9,12,15,18,21,24$ y 27 días de almacenamiento, con tres réplicas, para un total de 270 observaciones. Los datos obtenidos fueron analizados a través de un ANAVA con el uso del software estadístico Statistix 9, con un nivel de significancia del $5 \%$ y en caso de existir diferencias significativas entre los tratamientos se aplicó una prueba de rangos múltiples de Duncan.

\section{RESULTADOS Y DISCUSIÓN}

\section{pH}

El análisis de la varianza (ANAVA) muestra que el parámetro $\mathrm{pH}$ fue afectado significativamente por la interacción Día*recubrimiento $(\mathrm{p}<0,05)$, observándose que a medida que transcurría el tiempo de almacenamiento los valores mostraron una ligera tendencia a la disminución. En la tabla 1 se reportan los valores promedios, donde se observa que todas las muestras durante el periodo de almacenamiento tuvieron una disminución del $\mathrm{pH}$, siendo la muestra con recubrimiento comestible de $7 \%$ de almidón y glicerina la que menos cambios presentó durante los treinta días de almacenamiento, seguidas de las muestras de 5\% de almidón y glicerina, y la muestra con $0 \%$.

Tabla 1. Efecto del tiempo-recubrimientos sobre el parámetro $\mathrm{pH}$ en cascos de guayaba con recubrimientos comestibles a base de almidón de yuca y glicerina almacenados en refrigeración a $8{ }^{\circ} \mathrm{C}$

\begin{tabular}{|c|c|c|c|}
\hline \multirow{2}{*}{ Tiempo (Días) } & \multicolumn{3}{|c|}{ Recubrimientos Comestibles (RC) Almidón de yuca. } \\
\cline { 2 - 4 } & $0 \%$ & $5 \%$ & $7 \%$ \\
\hline 0 & $3,9678 \mathrm{~A}$ & $3,7389 \mathrm{C}$ & $3,7833 \mathrm{~B}$ \\
\hline 3 & $3,4344 \mathrm{I}$ & $3,5333 \mathrm{G}$ & $3,6833 \mathrm{D}$ \\
\hline 6 & $3,5078 \mathrm{H}$ & $3,3911 \mathrm{~J}$ & $3,5144 \mathrm{GH}$ \\
\hline 9 & $3,5389 \mathrm{G}$ & $3,2356 \mathrm{M}$ & $3,6944 \mathrm{D}$ \\
\hline 12 & $3,5378 \mathrm{G}$ & $3,6378 \mathrm{E}$ & $3,5689 \mathrm{~F}$ \\
\hline 15 & 3,14330 & $3,3467 \mathrm{~K}$ & $3,3967 \mathrm{~J}$ \\
\hline 18 & $2,9200 \mathrm{R}$ & $2,9067 \mathrm{R}$ & $3,2867 \mathrm{~L}$ \\
\hline 21 & $3,0733 \mathrm{Q}$ & $2,9233 \mathrm{R}$ & $3,3533 \mathrm{~K}$ \\
\hline 24 & $3,1067 \mathrm{P}$ & $3,1400 \mathrm{O}$ & $3,5033 \mathrm{H}$ \\
\hline 27 & $3,1833 \mathrm{~N}$ & $3,2433 \mathrm{M}$ & $3,4467 \mathrm{I}$ \\
\hline \multicolumn{2}{|c|}{ MDS= Letras diferentes indican promedios estadísticamente diferentes $(\mathrm{p} \leq 0,05)$} \\
\hline
\end{tabular}

\section{Cumbres}


El pH muestra un descenso a medida que transcurre el tiempo de almacenamiento en las tres formulaciones. El $\mathrm{pH}$ mantuvo un comportamiento constante en los primeros 9 días, notándose los cambios a partir del día 12. Sin embargo, a los 27 días de almacenamiento el $\mathrm{pH}$ se encontró cercano a lo establecido por la literatura, lo que quiere decir que los recubrimientos tuvieron un efecto en los cascos de guayaba. Desroiser (2002) reporta un pH para cascos de guayaba en un rango de 3,6 - 3,9 por lo que los resultados obtenidos se encuentran en este rango, siendo la muestra con recubrimiento comestible de $7 \%$ de almidón de yuca la que más se adecua a este parámetro.

El valor del pH varía en función del tiempo, a medida que la fruta continúa respirando aumenta su producción de etileno y se acelera su maduración, por lo tanto, el fruto produce varios ácidos, entre ellos el ascórbico, afectando el del pH (Wills et al., 1989). Investigaciones como las de Barco et al. (2009) que aplicaron un recubrimiento natural y cera comercial en bananos (Musa sapientum) obtuvieron resultados similares, donde el pH disminuyó progresivamente a través del tiempo de almacenamiento, pero los recubrimientos lograron relentecer este proceso alargando así el tiempo de vida útil de la fruta, y prolongando el sabor característico.

\section{Sólidos solubles, SS (Brix)}

El análisis de la varianza (ANAVA) muestra que el parámetro sólido soluble (SS) expresado en ํㅜix presentó efecto significativo para la interacción Día*recubrimiento $(\mathrm{p}<0,05)$, observándose que a medida en que transcurría el tiempo de almacenamiento los valores fueron mostrando una tendencia al aumento. En la tabla 2 se reportan los valores promedios donde se observa que todas las muestras durante el periodo de almacenamiento tuvieron un aumento de los SS, donde a partir del día 15 los SS se estabilizaron y permanecieron constantes para la formulación 3 (7\% de almidón de yuca). Los sólidos solubles deben incrementarse debido a la degradación hidrolítica de los carbohidratos de cadena larga; en donde es frecuente casi toda la conversión de almidón en azúcar, haciendo que el fruto sea más dulce (Aldana, 2001). El Instituto Nacional de nutrición (2001) en su tabla de composición de alimentos para uso práctico, estipula un rango de sólidos solubles para cascos de guayaba entre 8 - 11 oBrix.

Los recubrimientos comestibles son una barrera contra el proceso de respiración y, por tanto, la degradación del almidón en azúcares es más lenta, coincidiendo con lo reportado por Amaya et al. (2008) donde aplicaron un recubrimiento comestible de almidón de yuca nativo en tomates (Lycopersicom esculentum Mill) y por Rojas (2011) donde se usó un recubrimiento comestible y sustancias de origen natural en manzana fresca cortada, ambos trabajos presentaron comportamientos similares para el parámetro sólidos solubles.

En el día 15 la muestra de 5\% tuvo un aumento en los SS, podría ser que la fruta presentó una mayor producción de etileno por ende su maduración fue más rápida lo que produjo el aumento en los sólidos solubles. Para la muestra de $0 \%$ los SS se mantuvieron constantes desde el día 18 hasta finalizar el estudio al día 27 de almacenamiento. 
Tabla 2. Efecto del tiempo-recubrimientos sobre el parámetro Sólidos Solubles (SS) (ํㅜrix) en cascos de guayaba con recubrimientos comestibles a base de almidón de yuca y glicerina almacenados en refrigeración a $8 \stackrel{\circ}{\circ} \mathrm{C}$

\begin{tabular}{|c|c|c|c|}
\hline \multirow{2}{*}{ Tiempo (Días) } & \multicolumn{3}{|c|}{ Recubrimientos Comestibles (RC) Almidón de yuca. } \\
\cline { 2 - 4 } & $0 \%$ & $5 \%$ & $7 \%$ \\
\hline 0 & $7,333 \mathrm{~J}$ & $10,667 \mathrm{H}$ & $8,000 \mathrm{I}$ \\
\hline 3 & $13,333 \mathrm{D}$ & $13,333 \mathrm{D}$ & $13,333 \mathrm{D}$ \\
\hline 6 & $14,000 \mathrm{C}$ & $12,000 \mathrm{G}$ & $14,667 \mathrm{~B}$ \\
\hline 9 & $7,333 \mathrm{~J}$ & $10,667 \mathrm{H}$ & $6,667 \mathrm{~K}$ \\
\hline 12 & $7,333 \mathrm{~J}$ & $7,333 \mathrm{~J}$ & $12,667 \mathrm{~F}$ \\
\hline 15 & $13,333 \mathrm{D}$ & $18,667 \mathrm{~A}$ & $13,333 \mathrm{D}$ \\
\hline 18 & $8,000 \mathrm{I}$ & $12,000 \mathrm{G}$ & $13,333 \mathrm{D}$ \\
\hline 21 & $8,000 \mathrm{I}$ & $12,000 \mathrm{G}$ & $13,333 \mathrm{D}$ \\
\hline 24 & $8,000 \mathrm{I}$ & $13,103 \mathrm{E}$ & $13,333 \mathrm{D}$ \\
\hline 27 & $8,000 \mathrm{I}$ & $13,330 \mathrm{D}$ & $13,333 \mathrm{D}$ \\
\hline \multicolumn{2}{|c|}{ MDS= Letras diferentes indican promedios estadísticamente diferentes $(\mathrm{p} \leq 0,05)$} \\
\hline
\end{tabular}

El consumo de azúcares y ácidos orgánicos durante la respiración del fruto, la conversión de ácidos orgánicos en azúcares, la pérdida de agua por deshidratación, así como la hidrólisis de polisacáridos con la liberación de azúcares solubles propia del metabolismo del fruto (Barreiro et al., 2001), dan como resultado un balance negativo de ácidos orgánicos y una estabilización del contenido en azúcares que se traduce en un aumento y posterior estabilización de los sólidos solubles. Una clara muestra de lo expuesto es la investigación realizada, donde los SS aumentaron a medida que transcurría el tiempo de almacenamiento hasta llegar a un punto constante. Este aumento se debe a la degradación por reacciones de hidrólisis que sufre el almidón durante la maduración, convirtiéndose a azúcares más simples como sacarosa, fructosa y glucosa que genera un incremento en los sólidos solubles (Gallo, 1996).

\section{Acidez titulable (AT)}

En la tabla 3 se reportan los valores promedios, donde se observa que todas las muestras durante el periodo de almacenamiento tuvieron variación en la AT. Esto puede ser debido al cambio en el contenido de ácidos orgánicos en los frutos durante el almacenamiento, pero no se observó un efecto por el tratamiento ya que todos los frutos presentaron este comportamiento confiriendo a los cascos de guayaba con recubrimientos el mismo sabor característico de esta fruta fresca.

A pesar de ciertos picos que se presentaron en el día 15, la acidez titulable permaneció casi sin variación hasta finalizar el estudio. Wills (1999) estipula un rango de acidez titulable para cascos de guayaba entre $0,18-0,28$, por lo cual las tres muestras se ajustan a lo establecido en la literatura. El consumo de azúcares y ácidos orgánicos durante la respiración del fruto, la conversión de ácidos orgánicos en azúcares, la pérdida de agua por deshidratación, así como la hidrólisis de polisacáridos con la liberación de azucares solubles propia del metabolismo del fruto (Barreiro et al., 2001), dan como resultado un

\section{Cumbres}


balance negativo en la acidez titulable y una estabilización del contenido en azúcares que se traduce en una estabilización de los sólidos solubles.

Tabla 3. Efecto del tiempo-recubrimientos sobre el parámetro Acidez Titulable (AT) en cascos de guayaba con recubrimientos comestibles a base de almidón de yuca y glicerina almacenados en refrigeración a $8 \stackrel{\circ}{\circ} \mathrm{C}$

\begin{tabular}{|c|c|c|c|}
\hline \multirow{2}{*}{ Tiempo (Días) } & \multicolumn{3}{|c|}{ Recubrimientos Comestibles (RC) Almidón de yuca. } \\
\cline { 2 - 4 } & $0 \%$ & $5 \%$ & $7 \%$ \\
\hline 0 & $0,0690 \mathrm{G}$ & $0,0862 \mathrm{~F}$ & $0,0517 \mathrm{H}$ \\
\hline 3 & $0,1035 \mathrm{E}$ & $0,1035 \mathrm{E}$ & $0,1035 \mathrm{E}$ \\
\hline 6 & $0,1380 \mathrm{D}$ & $0,1380 \mathrm{E}$ & $0,0690 \mathrm{G}$ \\
\hline 9 & $0,1035 \mathrm{E}$ & $0,1552 \mathrm{C}$ & $0,1552 \mathrm{C}$ \\
\hline 12 & $0,1035 \mathrm{E}$ & $0,1035 \mathrm{E}$ & $0,1035 \mathrm{E}$ \\
\hline 15 & $0,2587 \mathrm{~A}$ & $0,2242 \mathrm{~B}$ & $0,1380 \mathrm{D}$ \\
\hline 18 & $0,1552 \mathrm{C}$ & $0,1552 \mathrm{C}$ & $0,1552 \mathrm{C}$ \\
\hline 21 & $0,1035 \mathrm{E}$ & $0,1035 \mathrm{E}$ & $0,1552 \mathrm{C}$ \\
\hline 24 & $0,1552 \mathrm{C}$ & $0,1380 \mathrm{D}$ & $0,1035 \mathrm{E}$ \\
\hline 27 & $0,1552 \mathrm{C}$ & $0,1035 \mathrm{E}$ & $0,1035 \mathrm{E}$ \\
\hline \multicolumn{2}{|c|}{ MDS $=$ Letras diferentes indican promedios estadísticamente diferentes $(\mathrm{p} \leq 0,05)$} \\
\hline
\end{tabular}

Al prolongar el almacenamiento hay una reducción de acidez ya que la fruta continúa respirando y produciendo etileno, por lo que su maduración la lleva a un aumento en los sólidos solubles, es decir, hay una relación entre los efectos de maduración-acidez-sólidos solubles. En estudio realizado por Zambrano y Briceño (2011) donde recubrieron mangos con ceras artificiales se observó diferencias en el contenido de acidez de los frutos tratados, situación que coincide en lo planteado en esta investigación, caso opuesto sucedió en lo planteado por Barco et al. (2009) donde la AT disminuyó progresivamente en los bananos (Musa sapientum) recubiertos con cera comercial a medida que transcurría el tiempo de almacenamiento, dado que durante la maduración, los ácidos orgánicos son convertidos en azúcares, por consiguiente, se espera que su contenido decline (Angón, 2010).

\section{Vitamina C (\% de ácido ascórbico)}

En la Tabla 4 se observa que todas las muestras durante el periodo de almacenamiento tuvieron una disminución en el contenido de vitamina C, este parámetro se mantuvo constante los primeros 9 días para los tres tratamientos, la pérdida empezó a mostrarse a partir del día 15 donde la formulación con $5 \%$ de almidón de yuca fue la que mayor pérdida, seguida por la de $7 \%$ y finalmente la de $0 \%$.

La muestra con recubrimiento comestible de 5\% de almidón de yuca y glicerina mostró la mayor pérdida de ácido ascórbico, la menor pérdida ocurrió en la muestra de $0 \%$ de almidón de yuca, a partir del día 24 las tres muestras se estabilizaron hasta finalizar el estudio.

Restrepo et al. (2007) reportaron valores del contenido de ácido ascórbico para guayaba de 74,77 - 111,86 expresados en $\mathrm{mg} / 100 \mathrm{~g}$, los valores obtenidos en el estudio se encuentran por debajo de lo establecido por la literatura 
pero no se atribuye al efecto de los recubrimientos sino a la manipulación pretratamiento, ya que las frutas fueron sometidas a un proceso de pelado y troceado antes de ser sometidas a inmersión en los recubrimientos comestibles, posterior a esto fueron sometidas a secado en una estufa de aire forzado para ayudar a la adhesión del recubrimiento, lo que implica una pérdida significativa de vitamina $\mathrm{C}$.

Tabla 4. Efecto del tiempo-recubrimientos sobre el parámetro Vitamina C (\% de ácido ascórbico) en cascos de guayaba con recubrimientos comestibles a base de almidón de yuca y glicerina almacenados en refrigeración a $8 \stackrel{\circ}{\circ}$

\begin{tabular}{|c|c|c|c|}
\hline \multirow{2}{*}{ Tiempo (Días) } & \multicolumn{3}{|c|}{ Recubrimientos Comestibles (RC) Almidón de yuca. } \\
\cline { 2 - 4 } & $0 \%$ & $5 \%$ & $7 \%$ \\
\hline 0 & $18,003 \mathrm{KL}$ & $19,503 \mathrm{JK}$ & $19,503 \mathrm{JK}$ \\
\hline 3 & $25,504 \mathrm{H}$ & $31,505 \mathrm{EF}$ & $33,005 \mathrm{E}$ \\
\hline 6 & $30,004 \mathrm{FG}$ & $33,005 \mathrm{E}$ & $19,503 \mathrm{JK}$ \\
\hline 9 & $16,502 \mathrm{~L}$ & $28,504 \mathrm{G}$ & $21,003 \mathrm{JJ}$ \\
\hline 12 & $18,003 \mathrm{KL}$ & $22,503 \mathrm{I}$ & $28,504 \mathrm{G}$ \\
\hline 15 & $30,004 \mathrm{FG}$ & $46,507 \mathrm{~B}$ & $37,506 \mathrm{D}$ \\
\hline 18 & $18,003 \mathrm{KL}$ & $49,507 \mathrm{~A}$ & $33,005 \mathrm{E}$ \\
\hline 21 & $13,502 \mathrm{M}$ & $45,007 \mathrm{~B}$ & $36,005 \mathrm{D}$ \\
\hline 24 & $33,005 \mathrm{E}$ & $40,506 \mathrm{C}$ & $31,505 \mathrm{EF}$ \\
\hline 27 & $36,005 \mathrm{D}$ & $36,005 \mathrm{D}$ & $36,005 \mathrm{D}$ \\
\hline \multicolumn{2}{|c|}{} \\
\hline \multicolumn{2}{|c|}{} \\
\hline
\end{tabular}

A partir del día 15 de almacenamiento, se observa que el recubrimiento comestible de 5 y 7\% de almidón de yuca y glicerina conservan el contenido de ácido ascórbico en los cascos de guayaba. La muestra con $0 \%$ de almidón de yuca continuó perdiendo vitamina C durante el almacenamiento. Valores similares fueron encontrados por Islam et al. (1993) en muestras de papaya y por De souza et al. (2008) en papaya del grupo "Solo" cv. "Golden" tratadas con recubrimientos comestibles a base de almidón de yuca al 2\%. Sin embargo, Adetuyi et al. (2008) obtuvieron valores mayores en muestras de papaya cultivadas en Nigeria recubiertas con manteca derretida de karité (Vitellarina paradoxa) almacenadas en refrigeración a $10 \stackrel{\circ}{\circ}$.

\section{EVALUACIÓN SENSORIAL}

\section{Aroma}

Este parámetro, el cual fue evaluado a través de un panel semientrenado, mostró un comportamiento uniforme en las muestras de 5 y 7\% de almidón de yuca hasta el día 12 posterior a este día de almacenamiento los tres tratamientos tuvieron un descenso considerable, más notorio para la muestra de 0\% de almidón de yuca (tabla 5).

La puntuación por parte de los panelistas, al recubrimiento comestible de $7 \%$ de almidón de yuca aplicado a los cascos de guayaba fue el óptimo, ya que el atributo aroma se mantuvo constante en esta muestra durante los días de almacenamiento mostrando una disminución a partir del día 24, lo que se

\section{Cumbres}


traduce en que el aroma característico de esta fruta se mantuvo intacto a diferencia de los otros 2 tratamientos que muestran un descenso a partir del día 9 de almacenamiento.

Tabla 5. Efecto del tiempo-recubrimientos sobre el parámetro sensorial Aroma en cascos de guayaba con recubrimientos comestibles a base de almidón de yuca y glicerina almacenados en refrigeración a $8 \stackrel{\circ}{\circ}$

\begin{tabular}{|c|c|c|c|}
\hline \multirow{2}{*}{ Tiempo (Días) } & \multicolumn{3}{|c|}{ Recubrimientos Comestibles (RC) Almidón de yuca. } \\
\cline { 2 - 4 } & $0 \%$ & $5 \%$ & $7 \%$ \\
\hline 0 & $5,0000 \mathrm{~A}$ & $5,0000 \mathrm{~A}$ & $5,0000 \mathrm{~A}$ \\
\hline 3 & $5,0000 \mathrm{~A}$ & $5,0000 \mathrm{~A}$ & $5,0000 \mathrm{~A}$ \\
\hline 6 & $4,0000 \mathrm{~B}$ & $5,0000 \mathrm{~A}$ & $5,0000 \mathrm{~A}$ \\
\hline 9 & $4,0000 \mathrm{~B}$ & $5,0000 \mathrm{~A}$ & $5,0000 \mathrm{~A}$ \\
\hline 12 & $2,0000 \mathrm{E}$ & $5,0000 \mathrm{~A}$ & $5,0000 \mathrm{~A}$ \\
\hline 15 & $2,0000 \mathrm{E}$ & $3,0000 \mathrm{D}$ & $5,0000 \mathrm{~A}$ \\
\hline 18 & $2,0000 \mathrm{E}$ & $3,0000 \mathrm{D}$ & $5,0000 \mathrm{~A}$ \\
\hline 21 & $2,0000 \mathrm{E}$ & $1,0000 \mathrm{~F}$ & $5,0000 \mathrm{~A}$ \\
\hline 24 & $2,0000 \mathrm{E}$ & $1,0000 \mathrm{~F}$ & $4,0000 \mathrm{~B}$ \\
\hline 27 & $1,0000 \mathrm{~F}$ & $1,0000 \mathrm{~F}$ & $3,5833 \mathrm{C}$ \\
\hline MDS $=$ Letras diferentes indican promedios estadísticamente diferentes $(\mathrm{p} \leq 0,05)$ \\
\hline
\end{tabular}

Los recubrimientos comestibles se comportan como barrera semipermeable que reduce el paso de compuestos aromáticos desprendidos por el fruto (Han y Leonard, 2004), mejorando en los panelistas la percepción del aroma.

El recubrimiento comestible con mayor concentración de almidón de yuca formó esta barrera semipermeable que redujo el paso de compuestos aromáticos, tomando en cuenta que en estudios posteriores a esta fruta, el aroma se veía alterado a partir del día 9 de almacenamiento, lo que quiere decir que el recubrimiento resguarda el aroma característico de la guayaba hasta por 2 semanas más de lo establecido para la investigación.

Giménez (2003) indicó que las bajas temperaturas influyen en la disminución del aroma, sin embargo, a pesar que las muestras estudiadas fueron almacenadas a bajas temperaturas $\left({ }^{\circ} \stackrel{\circ}{ } \mathrm{C}\right)$, los panelistas no percibieron malos olores en los cascos de guayaba con los diferentes recubrimientos comestibles.

El aroma de la guayaba cultivar cubana suprema roja fue estudiado por Steinhaus et al. (2008), quienes catalogaron el aroma de la guayaba como intenso y agradable, y lo detectaron como notas olfativas grasosa, "grapefruit", frutal, fresco, metálico, dulce - caramelo y floral, debido principalmente a la presencia de compuestos azufrados, estos resultados son similares a los encontrados en esta investigación, a pesar de que en la evaluación no se estudiaron, ni lograron identificar los compuestos volátiles responsables del aroma de la guayaba.

\section{Consistencia}

Los valores promedios se reportan en la tabla 6, donde se observa que todas las muestras durante el periodo de almacenamiento tuvieron una disminu- 
ción de la consistencia, este parámetro, el cual fue evaluado a través de un panel semientrenado, mostró un comportamiento uniforme en las muestras de $5 \%$ de almidón de yuca hasta el día 12 , posterior a este día de almacenamiento los tres tratamientos tuvieron un decline considerable, más notorio para la muestra de 5\% de almidón de yuca, el tratamiento con 7\% de almidón se mantuvo constante hasta el día 21 de almacenamiento.

Tabla 6. Efecto del tiempo-recubrimientos sobre el parámetro sensorial Consistencia en cascos de guayaba con recubrimientos comestibles a base de almidón de yuca y glicerina almacenados en refrigeración a $8 \stackrel{\circ}{\circ}$

\begin{tabular}{|c|c|c|c|}
\hline \multirow{2}{*}{ Tiempo (Días) } & \multicolumn{3}{|c|}{ Recubrimientos Comestibles (RC) Almidón de yuca. } \\
\cline { 2 - 4 } & $0 \%$ & $5 \%$ & $7 \%$ \\
\hline 0 & $5,0000 \mathrm{~A}$ & $5,0000 \mathrm{~A}$ & $5,0000 \mathrm{~A}$ \\
\hline 3 & $5,0000 \mathrm{~A}$ & $5,0000 \mathrm{~A}$ & $5,0000 \mathrm{~A}$ \\
\hline 6 & $4,0000 \mathrm{~B}$ & $5,0000 \mathrm{~A}$ & $5,0000 \mathrm{~A}$ \\
\hline 9 & $4,0000 \mathrm{~B}$ & $5,0000 \mathrm{~A}$ & $5,0000 \mathrm{~A}$ \\
\hline 12 & $2,0000 \mathrm{E}$ & $5,0000 \mathrm{~A}$ & $5,0000 \mathrm{~A}$ \\
\hline 15 & $2,0000 \mathrm{E}$ & $3,0000 \mathrm{D}$ & $5,0000 \mathrm{~A}$ \\
\hline 18 & $2,0000 \mathrm{E}$ & $3,0000 \mathrm{D}$ & $5,0000 \mathrm{~A}$ \\
\hline 21 & $2,0000 \mathrm{E}$ & $1,0000 \mathrm{G}$ & $5,0000 \mathrm{~A}$ \\
\hline 24 & $2,0000 \mathrm{E}$ & $1,0000 \mathrm{G}$ & $4,0000 \mathrm{~B}$ \\
\hline 27 & $1,3333 \mathrm{~F}$ & $1,0000 \mathrm{G}$ & $3,5833 \mathrm{C}$ \\
\hline \multicolumn{2}{|c|}{ MDS= Letras diferentes indican promedios estadísticamente diferentes $(\mathrm{p} \leq 0,05)$} \\
\hline
\end{tabular}

Se puede observar como el parámetro sensorial consistencia fue disminuyendo a medida que transcurría el tiempo de almacenamiento, siendo las muestras de $0 \%$ y $5 \%$ de almidón de yuca las que sufrieron en cuanto a textura, es decir la pérdida de turgencia por efecto de la senescencia del fruto. Evidenciando que el recubrimiento comestible de 7\% de almidón de yuca fue la más efectiva, prolongando por mayor tiempo la consistencia de los cascos de guayaba.

La textura de los cascos de guayaba es un factor importante en la calidad de los mismos y limita su período de almacenamiento. En la experiencia realizada, los panelistas detectaron una pérdida de consistencia al prolongar el almacenamiento sobre todo en las muestras recubiertas con baja concentración de almidón de yuca, la consistencia fue aceptable para estas muestras hasta el día 12; sin embargo, caso diferente fue para la muestra de $7 \%$ que su textura óptima se mantuvo hasta el día 24 de almacenamiento.

\section{Sabor}

En la tabla 7 se observa que todas las muestras durante el periodo de almacenamiento tuvieron una disminución del sabor y mostró un comportamiento uniforme en las muestras de 5 y $7 \%$ de almidón de yuca hasta el día 18, posterior a este día de almacenamiento los tres tratamientos tuvieron un descenso considerable, más notorio para la muestra de 0 y $5 \%$ de almidón de yuca, las cuales se comportaron de manera similar a partir del día 21 de almacenamiento hasta finalizar el estudio.

\section{Cumbres}


Se observa que ocurre un decline en el parámetro sensorial sabor en las muestras recubiertas con 0 y $5 \%$ de almidón de yuca, caso contrario muestran los cascos de guayaba recubiertos con $7 \%$, donde su sabor permanece constante hasta el día 21 de almacenamiento mostrando una ligera disminución en los días posteriores, ratificando así que este recubrimiento ayudó a mantener las propiedades organolépticas del producto.

Tabla 6. Efecto del tiempo-recubrimientos sobre el parámetro sensorial Consistencia en cascos de guayaba con recubrimientos comestibles a base de almidón de yuca y glicerina almacenados en refrigeración a $8 \stackrel{\circ}{\circ}$

\begin{tabular}{|c|c|c|c|}
\hline \multirow{2}{*}{ Tiempo (Días) } & \multicolumn{3}{|c|}{ Recubrimientos Comestibles (RC) Almidón de yuca. } \\
\cline { 2 - 4 } & $0 \%$ & $5 \%$ & $7 \%$ \\
\hline 0 & $5,0000 \mathrm{~A}$ & $5,0000 \mathrm{~A}$ & $5,0000 \mathrm{~A}$ \\
\hline 3 & $5,0000 \mathrm{~A}$ & $5,0000 \mathrm{~A}$ & $5,0000 \mathrm{~A}$ \\
\hline 6 & $4,0000 \mathrm{~B}$ & $5,0000 \mathrm{~A}$ & $5,0000 \mathrm{~A}$ \\
\hline 9 & $4,0000 \mathrm{~B}$ & $5,0000 \mathrm{~A}$ & $5,0000 \mathrm{~A}$ \\
\hline 12 & $2,0000 \mathrm{E}$ & $5,0000 \mathrm{~A}$ & $5,0000 \mathrm{~A}$ \\
\hline 15 & $2,0000 \mathrm{E}$ & $3,0000 \mathrm{D}$ & $5,0000 \mathrm{~A}$ \\
\hline 18 & $2,0000 \mathrm{E}$ & $3,0000 \mathrm{D}$ & $5,0000 \mathrm{~A}$ \\
\hline 21 & $1,0000 \mathrm{~F}$ & $1,0000 \mathrm{~F}$ & $5,0000 \mathrm{~A}$ \\
\hline 24 & $1,0000 \mathrm{~F}$ & $1,0000 \mathrm{~F}$ & $4,0000 \mathrm{~B}$ \\
\hline 27 & $1,0000 \mathrm{~F}$ & $1,0000 \mathrm{~F}$ & $3,5833 \mathrm{C}$ \\
\hline MDS= Letras diferentes indican promedios estadísticamente diferentes $(\mathrm{p} \leq 0,05)$ \\
\hline
\end{tabular}

Cuando los recubrimientos comestibles ejercen una excesiva barrera a los gases $\mathrm{CO}_{2}$ y $\mathrm{O}_{2}$, aumentan los niveles de etanol y acetaldehído del fruto y disminuye la calidad organoléptica de los mismos (Cohen et al., 1990), alterando el sabor característico de los frutos. El recubrimiento comestible elaborado con $7 \%$ de almidón de yuca y 5\% de glicerina mantuvo un equilibrio en estos gases permitiendo la conservación de las propiedades sensoriales de los cascos de guayaba sometidos a estudio durante 27 días de almacenamiento en refrigeración.

Para que un recubrimiento comestible sea óptimo debe ser incoloro que no adjudique un aroma o sabor más fuerte o diferente al característico del alimento al natural, como lo exponen Famá et al. (2009) quienes estudiaron nanocompuestos biodegradables y comestibles a base de almidón y polvo de ajo; llegando a la conclusión de que esta combinación alteraba significativamente las características organolépticas sobre todo en el aroma y sabor, en consecuencia se presentaba un rechazo por parte del consumidor.

\section{CONCLUSIONES}

Los sólidos solubles aumentaron en todo el periodo de almacenamiento (27 días). El pH, acidez titulable y porcentaje de ácido ascórbico no se vieron afectados por los recubrimientos, teniendo un comportamiento decreciente en las tres formulaciones, pero adjudicado a las condiciones de maduración del fruto, el tiempo de almacenamiento y la manipulación pretratamiento. El recubrimiento comestible con $7 \%$ de almidón de yuca y glicerina no modificó 
los atributos sensoriales estudiados (aroma, consistencia y sabor), por el contrario, ayudó a preservar las características originales de la fruta por más tiempo, conservando así su aroma, sabor y consistencia.

\section{REFERENCIAS BIBLIOGRÁFICAS}

ADETUYI, F.; AKINADEWO, L.; OMOSULI S. y AJALA, L. 2008. Antinutrient and antioxidant quality of waxed and unwaxed pawpaw (Carica papaya) fruit stored at different temperatures. African Journal of Biotechnology. 7(16):2920-2924.

ALDANA, H. 2001. Ingeniería y agroindustria. Continental S.A. Bogotá. 147$153 \mathrm{p}$.

ALEIXANDRE, J. 1987. Procesos generales de conservación y envasado de alimentos. Servicio de publicaciones Universidad Politécnica de Valencia. $353 \mathrm{p}$.

AMAYA, P.; PEÑA, L. y MOSQUERA, A. 2008. Efecto de recubrimiento de almidón nativo de yuca sobre la firmeza y tasa de respiración del tomate (Lycopersicom esculentum mill). Trabajo de grado de Ingeniería Agroindustrial. Popayán: Universidad del Cauca. Facultad de Ciencias Agropecuarias. Departamento de Agroindustria. 115 p.

BARCO, P.; BURABANO, A.; MEDINA, M.; MOSQUERA, S. y VILLADA, H. 2009. Efecto de recubrimiento natural y cera comercial sobre la maduración de banano (Musa sapientum). Trabajo de grado de la facultad de ciencias agropecuarias. Universidad del Cauca. Popayán.

BARREIRO, M.; CEBOLA, F. y PINTO, M. 2001. Physicochemical characterisation of the postharvest senescence of winter melon 'Tendral'. Fruits, 56:51-58.

CARRASCO, E.; VILLARUEL, M. y CISNEROS, L. 2002. Efectos de recubrimientos comestibles sobre la calidad sensorial de pimentones verdes (Capsicum annuum L.) durante el almacenamiento. Temuco. Chile. 82-95p

COHEN, E.; SHALOM, Y. y ROSENBERG, I. 1990. Postharvest ethanol buildup and off-flavor in 'Murcot' tangerine fruits. J. Am. Hort. Soc., 115:775778.

COVENIN. Comisión Venezolana De Normas Industriales.1977. Frutas y productos derivados. Determinación de la acidez. Norma venezolana № 1151 - 77. FONDONORMA. Caracas. Venezuela. 6p.

COVENIN. Comisión Venezolana De Normas Industriales. 1983. Frutas y productos derivados. Determinación de sólidos solubles por refractometría. Norma Venezolana № 924-83. FONDONORMA. Caracas. Venezuela. 2p.

DESROISER, N. 2002. Conservación de los alimentos. Continental S.A., Ciudad de México, p.22-23

DE SOUZA, L.; FERREIRA, K.; CHAVES, JOSÉ. y TEIXEIRA, S. 2008. L-ascorbic acid, $\beta$-carotene and lycopene content in papaya fruits (Carica papaya) with or without physiological skin freckles. Scientia Agricola. 65(3):246250.

\section{Cumbres}


FAMÁ L. y GOYANES, S. 2008 Estudio de las propiedades fisicoquímicas de recubrimientos compuestos comestibles y biodegradables, Tesis Ph.D. Buenos Aires (Argentina): Facultad de Ciencias Exactas y Naturales, Universidad de Buenos Aires.

FAMÁ L.; GERSCHENSON, L. y GOYANES, S. 2009. Nanocompuestos biodegradables y comestibles: Almidón-Polvo de ajo. Suplemento de la revista Latinoamericana de Metalurgia y Materiales. Universidad Simón Bolívar. Venezuela.

GALLO, F. 1996. Manual de fisiología, patología post-cosecha y control de calidad de frutas y hortalizas. Colombia. 25 - 32p.

GIMÉNEZ, E. 2003. Innovaciones tecnológicas en la conservación de melón y tomate procesado en fresco. Tesis doctoral. Universidad Politécnica de Cartagena. Departamento de ingeniería de alimentos y del equipamiento agrícola. Colombia. 67-78 p.

HAN, C.; ZHAO, Y.; LCONRAD, S. y TRABER, M. 2004. Edible coatings to improve storability and enhance nutritional value of fresh and frozen strawberries (Fragaria x ananassa) and raspberries (Rubus ideaus). Postharv. Biol. Technol., 33:67-78.

INSTITUTO NACIONAL DE NUTRICIÓN. 2001. Tabla de Composición de Alimentos para Uso Práctico. Publicación No. 54. Serie Cuadernos Azules. Caracas. Venezuela. 84 p.

ISLAM, N.; COLÓN, T. y VARGAS, T. 1993. Effect of prolonged solar exposure on the vitamin $\mathrm{C}$ contents of tropical fruits. Food Chemistry. 48(1):75-78.

MATA, B. y RODRÍGUEZ, A. 1990. Cultivo y producción del guayabo. TRILLAS. México. 55-90 p.

MARTIN, M.; MAUGUIN, C. y VOLLEY, A. 1992. Hydrophobic films and their efficiency against moisture transfer. 1. Influence of the film preparation technique. J. Agric. Food Chem. 40: 407-412p.

McHUGH, T. y SENESI, E. 2000. Apple wraps: a novel method to improve the quality and extend the shelf life of fresh-cut apples. J. Food Sci. 65: 480-485p.

MILLER, D. 2003. Química de los alimentos. Manual de laboratorio. Limusa Wiley. Df, México. 173p.

RESTREPO D.; NARVÁES C. y RESTREPO L. 2007. Actividad antioxidante y fenoles en guayaba de Colombia. Bogotá-Colombia. 54-56 p.

ROJAS, M. 2011. Recubrimientos comestibles y sustancias de origen natural en manzana fresca cortada: una nueva estrategia de conservación. (Documento en línea). Disponible en: http://biblioteca.universia.net/htmL bura/ficha/params/id/22585912.htmL. Consultado [15/01/2012].

ROMOJARO, F.; REQUELME, F.; PRETEL, M.; MARTINEZ, G.; SERRANO, S.; MARTINEZ, C.; LOZANO, P.; SEQUERA, P. y LUNA, P. 1996.

Nuevas tecnologías de conservación de frutas y hortalizas. Atmosferas Modificadas. Mundi-Prensa. Madrid, España. 221 p.

SCOTT, K. y WILLS, R. 1979. Effects of vacuum and pressure infiltration of calcium chloride and storage temperature on the incidence of bitter pit and low temperature breakdown of apples. Journal of Agricultural Research, 30: 917-928. 
STEINHAUS, M.; SINUCO, D.; POLSTER, J.; OSORIO, C. y SCHIEBERLE, P. 2008. Characterization of the aroma-active compounds in Pink Guava (Psidium guajava L.) by application of the aroma extract dilution analysis. Journal of Agriculture Food Chemistry. 56, 4120-4127.

VICENTINI, N.; ROSSETO, T. y CERDA, M. 1999. Influencia de fécula de mandioca en las cualidades postcosecha de frutos de pimentón (Capsicum annuum L.). Capinas Jan./ Apr. 1-5p.

WILLS, R.; MAGLASON, B.; GRAHAM, D. y JOYCED, L. 1999. Introducción a la Fisiología y manipulación Postcosecha de frutas y hortalizas y plantas ornamentales. Acribia, Zaragoza, España. 